Kennesaw State University

DigitalCommons@Kennesaw State University

Faculty Publications

$2-2012$

\title{
The Kolmogorov and Stechkin Problems for Classes of Functions Whose Second Derivative Belongs to the Orlicz Space
}

Yuliya Babenko

Kennesaw State University, ybabenko@kennesaw.edu

Dmitry Skorokhodov

Dnepropetrovsk National University, Dnepropetrovsk Russia

Follow this and additional works at: http://digitalcommons.kennesaw.edu/facpubs

Part of the Analysis Commons

\section{Recommended Citation}

Babenko, Y., \& Skorokhodov, D. (2012). The kolmogorov and stechkin problems for classes of functions whose second derivative belongs to the orlicz space. Mathematical Notes, 91(1), 161-171. doi:10.1134/S000143461201018X

This Article is brought to you for free and open access by DigitalCommons@Kennesaw State University. It has been accepted for inclusion in Faculty Publications by an authorized administrator of DigitalCommons@Kennesaw State University. For more information, please contact 


\title{
The Kolmogorov and Stechkin Problems for Classes of Functions Whose Second Derivative Belongs to the Orlicz Space
}

\author{
Yu. V. Babenko ${ }^{1 *}$ and D. S. Skorokhodov ${ }^{2 * *}$ \\ ${ }^{1}$ Kennesaw State University, USA \\ ${ }^{2}$ Dnepropetrousk National University \\ Received December 10, 2009; in final form, February 27, 2010
}

\begin{abstract}
For any $t \in[0,1]$, we obtain the exact value of the modulus of continuity$$
\omega_{N}\left(D_{t}, \delta\right):=\sup \left\{\left|x^{\prime}(t)\right|:\|x\|_{L_{\infty}[0,1]} \leq \delta,\left\|x^{\prime \prime}\right\|_{L_{N}^{*}[0,1]} \leq 1\right\},
$$

where $L_{N}^{*}$ is the dual Orlicz space with Luxemburg norm and $D_{t}$ is the operator of differentition at the point $t$. As an application, we state necessary and sufficient conditions in the Kolmogorov problem for three numbers. Also we solve the Stechkin problem, i.e., the problem of approximating an unbounded operator of differentition $D_{t}$ by bounded linear operators for the class of functions $x$ such that $\left\|x^{\prime \prime}\right\|_{L_{N}^{*}[0,1]} \leq 1$.
\end{abstract}

\section{DOI: $10.1134 / \mathrm{S} 000143461201018 \mathrm{X}$}

Keywords: Kolmogorov problem for three numbers, Stechkin problem, Orlicz space, Luxemburg norm, operator of differentition, Banach space, modulus of continuity.

\section{INTRODUCTION}

As is customary, let $L_{s}:=L_{s}[0,1], 1 \leq s \leq \infty$, denote the spaces of functions $x:[0,1] \rightarrow \mathbb{R}$, measurable and $s$ th summable on $[0,1]$ (essentially bounded at $s=\infty$ ) with the corresponding norms

$$
\|x\|_{s}:= \begin{cases}\left(\int_{0}^{1}|x(t)|^{s} d t\right)^{1 / s}, & 1 \leq s<\infty \\ \operatorname{esssup}\{|x(t)|: t \in[0,1]\}, & s=\infty .\end{cases}
$$

Along with the spaces $L_{s}$, we also consider more general Orlicz spaces (see, for example, [1]). Let us present the required notation.

Let $p:[0, \infty) \rightarrow \mathbb{R}$ and $q:[0, \infty) \rightarrow \mathbb{R}$ be two right-continuous nondecreasing functions such that

$$
q(\rho):=\sup _{p(\tau) \leq \rho} \tau, \quad p(\tau):=\sup _{q(\rho) \leq \tau} \rho, \quad p(0)=q(0)=0, \quad p(\infty)=q(\infty)=\infty .
$$

Convex functions $M: \mathbb{R} \rightarrow \mathbb{R}$ and $N: \mathbb{R} \rightarrow \mathbb{R}$ defined by the equalities

$$
M(u)=\int_{0}^{|u|} p(\tau) d \tau, \quad N(v)=\int_{0}^{|v|} q(\tau) d \tau,
$$

are called complementary $N$-functions.

*E-mail: ybabenko@kennesaw.edu

${ }^{* *}$ E-mail: dmitriy.skorokhodov@gmail.com 
Suppose that $M(u)$ and $N(v)$ are complementary $N$-functions. Let $L_{M}:=L_{M}[0,1]$ denote the Orlicz class corresponding to the $N$-function $M(u)$, i.e., the class of functions $x:[0,1] \rightarrow \mathbb{R}$ for which

$$
\rho(x, M):=\int_{0}^{1} M(|x(\tau)|) d \tau<\infty .
$$

Let $L_{M}^{*}:=L_{M}^{*}[0,1]$ denote the class of functions $x:[0,1] \rightarrow \mathbb{R}$ such that

$$
\int_{0}^{1} x(\tau) y(\tau) d \tau<\infty \quad \text { for any } y \in L_{N} .
$$

Following the book [1, Sec. 9], for the class $L_{M}^{*}$ we introduce the Orlicz norm

$$
\|x\|_{M}:=\sup _{\rho(y, N) \leq 1}\left|\int_{0}^{1} x(\tau) y(\tau) d \tau\right|
$$

and the Luxemburg norm

$$
\|x\|_{(M)}:=\inf \left\{k>0: \rho\left(\frac{x}{k}, M\right) \leq 1\right\}
$$

Note that if $M(u)=u^{s}, 1<s<\infty$, then the class $L_{M}^{*}$ is exactly the class $L_{s}$, and the Luxemburg norm $\|\cdot\|_{(M)}$ coincides with the norm $\|\cdot\|_{s}$. In this connection, we note that Orlicz spaces are generalizations of the classes $L_{s}$.

Let $L_{M}^{*, r}, r \in \mathbb{N}$, denote the space of functions $x:[0,1] \rightarrow \mathbb{R}$ whose derivative $x^{(r-1)}$ is absolutely continuous and $x \in L_{M}^{*}$. In the case $M(u)=u^{s}, 1<s<\infty$, we set $L_{s}^{r}:=L_{M}^{*, r}$.

Let $1 \leq k \leq r-1$. The problem of sharp additive inequalities of the form

$$
\left|x^{(k)}(t)\right| \leq A\|x\|_{\infty}+B\left\|x^{(r)}\right\|_{s}
$$

for the function classes $L_{s}^{r}$ was studied in Landau's paper [2], where all pairs of positive numbers $(A, B)$ for which the inequality of the form (1.1) is sharp was characterized for $k=1, r=2$, and $s=\infty$. In what follows, inequalities (1.1) were studied in papers of many mathematicians. Some familiar results in this direction as well as a discussion of relevant questions can be found, for example, in the books [3], [4], and [5]. A result due to Pinkus [6] is at present the unique complete result on inequalities of the form (1.1). In the other cases, the theory is far from complete.

Along with the classes $L_{s}^{2}$, it is also of interest to study more general function classes, for example, the classes $L_{N}^{*, 2}$. Thus, in [7], for any fixed $t \in[0,1]$, the following sharp inequalities were obtained:

$$
\left|x^{\prime}(t)\right| \leq A\|x\|_{\infty}+B\left\|x^{\prime \prime}\right\|_{(N)},
$$

where $x \in L_{N}^{*, 2}$ and $N(v)$ is an $N$-function.

To state the corresponding result of [7], let us consider the following family of functions. For $t \in[0,1 / 2]$ and $h \in[0,1]$, we set

$$
g_{t, h}(\tau):= \begin{cases}0, & \tau \in\left[0, t-\frac{h}{2}\right], \\ \frac{1}{h}\left(t-\frac{h}{2}-\tau\right), & \tau \in\left[t-\frac{h}{2}, t\right], \\ \frac{1}{h}\left(t+\frac{h}{2}-\tau\right), & \tau \in\left[t, t+\frac{h}{2}\right], \\ 0, & \tau \in\left[t+\frac{h}{2}, 1\right],\end{cases}
$$


if $0<h \leq 2 t$, and

$$
g_{t, h}(\tau):= \begin{cases}-\frac{\tau}{h}, & \tau \in[0, t], \\ \frac{h-\tau}{h}, & \tau \in[t, h] \\ 0, & \tau \in[h, 1]\end{cases}
$$

if $2 t \leq h \leq 1$.

Theorem 1. Suppose that $t \in[0,1 / 2]$ and $h \in(0,1]$. Also suppose that $M(u)$ and $N(v)$ are complementary $N$-functions. Then, for any function $x \in L_{N}^{*, 2}$, the following inequality holds:

$$
\left|x^{\prime}(t)\right| \leq \frac{2}{h}\|x\|_{\infty}+\left\|g_{t, h}\right\|_{M}\left\|x^{\prime \prime}\right\|_{(N)} .
$$

Remark 1. For the classes $L_{s}^{2}, 1 \leq s<\infty$, Theorem 1 was also independently proved in [8].

Note that inequality (1.5) is sharp if the right derivative $p(\tau)$ of the function $M(u)$ is continuous. Indeed, let us construct the extremal functions for this case. Suppose that $0 \leq t \leq 1 / 2$ and $h \in(0,1]$. As proved in [1, p. 108], the continuity of the function $p(\tau)$ ensures the existence of a number $\alpha_{h}>0$ such that

$$
\int_{0}^{1} N\left[p\left(\alpha_{h}\left|g_{t, h}(\tau)\right|\right)\right] d \tau=1
$$

Set

$$
x_{t, h}^{\prime \prime}(\nu):=p\left(\alpha_{h}\left|g_{t, h}(\nu)\right|\right) \operatorname{sign} g_{t, h}(\nu), \quad \nu \in[0,1] .
$$

Further,

$$
x_{t, h}^{\prime}(u):=\int_{h}^{u} x_{t, h}^{\prime \prime}(\nu) d \nu, \quad u \in[0,1] .
$$

Obviously $x_{t, h}^{\prime}(u)$ is nonpositive on $[0,1]$. Now, for $h \in(0,2 t]$ we take $t_{h}^{*}:=t$, and for $h \in[2 t, 1]$ we choose $t_{h}^{*} \in(0, h)$ so that

$$
\int_{0}^{t_{h}^{*}} x_{t, h}^{\prime}(u) d u=\int_{t_{h}^{*}}^{h} x_{t, h}^{\prime}(u) d u .
$$

Finally, we define the function $x_{t, h}$ as

$$
x_{t, h}(\tau):=\int_{t_{h}^{*}}^{\tau} x_{t, h}^{\prime}(u) d u, \quad \tau \in[0,1] .
$$

Now, to verify that the function $x_{t, h}$ is extremal, it suffices to use the following facts.

Lemma 1 ([1, p. 106]). Suppose that $x(\tau) \in L_{M}^{*}$ and there exists a $k$ such that

$$
\int_{0}^{1} N[p(k|x(\tau)|)] d \tau=1
$$

where $p(\tau)$ is the right derivative of the function $M(u)$. Then

$$
\|x\|_{M}=\int_{0}^{1} p(k|x(\tau)|)|x(\tau)| d \tau .
$$

Lemma $2\left(\left[1\right.\right.$, p. 96]). Suppose that $x(\tau) \in L_{N}^{*}$ and

$$
\int_{0}^{1} N\left[\frac{x(\tau)}{k_{0}}\right] d \tau=1
$$

Then $\|x\|_{(N)}=k_{0}$. 
As a consequence from Theorem 1, we obtain the following statement.

Theorem 2. For any function $x \in L_{N}^{*, 2}$ and any $h \in(0,1]$, the following inequality holds:

$$
\left\|x^{\prime}\right\|_{\infty} \leq \frac{2}{h}\|x\|_{\infty}+\left\|g_{0, h}\right\|_{M}\left\|x^{\prime \prime}\right\|_{(N)} .
$$

Proof of Theorem 2. In order to prove inequality (1.9), we use the notion of rearrangement of a function (see, for example, [9, Chap. 1]). Let us recall some definitions.

With the summable function $x(u)$ on the closed interval $[0,1]$ and the number $y \geq 0$ we associate the number

$$
m(x, y):=\mu\{u \in[0,1]:|x(u)|>y\},
$$

where $\mu(E)$ is Lebesgue measure on the closed interval $[0,1]$. This equality defines a nonincreasing right-continuous function $m(x, y)$ on $[0, \infty)$, called the distribution function for $x(u)$.

A decreasing rearrangement of a function $f(u)$ is defined by the equality

$$
r(x, u):=\inf \{y \in[0, \infty): m(x, y) \leq u\} .
$$

A detailed description of the properties of rearrangements is given, for example, in [9, Chap. 1]. But here we need the simple fact that, for any $N$-function $\Phi(v)$,

$$
\int_{0}^{1} \Phi(|x(u)|) d u=\int_{0}^{1} \Phi(r(x, u)) d u .
$$

To apply property (1.10), we note, as a preliminary, that, for $0<h \leq 2 t$,

$$
r\left(g_{t, h}, \tau\right)= \begin{cases}\frac{h-\tau}{2 h}, & \tau \in[0, h] \\ 0, & \tau \in[h, 1]\end{cases}
$$

and, for $2 t \leq h \leq 1$,

$$
r\left(g_{t, h}, \tau\right)= \begin{cases}\frac{h-t-\tau}{h}, & \tau \in[0, h-2 t], \\ \frac{h-\tau}{2 h}, & \tau \in[h-2 t, h], \\ 0, & \tau \in[h, 1] .\end{cases}
$$

Therefore, for any $\tau \in[0,1]$,

$$
r\left(g_{t, h}, \tau\right) \leq r\left(g_{0, h}, \tau\right) .
$$

In view of the monotonicity of the Orlicz norm, using the last inequality, we find that

$$
\left\|r\left(g_{t, h}, \cdot\right)\right\|_{M} \leq\left\|r\left(g_{0, h}, \cdot\right)\right\|_{M} .
$$

Hence, by the property of (1.10), we have

$$
\left\|g_{t, h}\right\|_{M} \leq\left\|g_{0, h}\right\|_{M}
$$

Therefore,

$$
\left\|x^{\prime}\right\|_{\infty}=\sup _{t \in[0,1]}\left|x^{\prime}(t)\right| \leq \frac{2}{h}\|x\|_{\infty}+\sup _{t \in[0,1]}\left\|g_{t, h}\right\|_{M}\left\|x^{\prime \prime}\right\|_{(N)}=\frac{2}{h}\|x\|_{\infty}+\left\|g_{0, h}\right\|_{M}\left\|x^{\prime \prime}\right\|_{(N)} .
$$




\section{STATEMENT OF THE PROBLEMS AND MAIN RESULTS}

Suppose that $M(u)$ and $N(v)$ are complementary $N$-functions. Let $W_{N}^{*, 2}$ denote the class of functions $x \in L_{N}^{*, 2}$ for which $\left\|x^{\prime \prime}\right\|_{(N)} \leq 1$, and let $D$ and $D_{t}, 0 \leq t \leq 1$, be the operator of differentition and the operator of differentition at the point $t$, respectively.

The present paper is a continuation of [7] and [10]. Here we consider the more general problem of evaluating the moduli of continuity of the operators $D$ and $D_{t}$ for the classes $W_{N}^{*, 2}$. The problem in question is to determine, for all $\delta>0$, the quantities

$$
\begin{aligned}
\omega_{N}(D, \delta) & :=\sup \left\{\left\|x^{\prime}\right\|_{\infty}: x \in W_{N}^{*, 2},\|x\|_{\infty} \leq \delta\right\}, \\
\omega_{N}\left(D_{t}, \delta\right) & :=\sup \left\{\left|x^{\prime}(t)\right|: x \in W_{N}^{*, 2},\|x\|_{\infty} \leq \delta\right\} .
\end{aligned}
$$

To state the results obtained, for all $h \geq 1$ we set

$$
x_{t, h}(\tau):=x_{t, 1}(\tau)+\left\|x_{t, 1}\right\|_{\infty}(h-1)\left(\frac{1}{2}-\tau\right), \quad \tau \in[0,1],
$$

where the function $x_{t, 1}(\tau)$ is defined by relation (1.7).

The following statements hold.

Theorem 3. Suppose that $M(u)$ and $N(v)$ are complementary $N$-functions and the right derivative $p(\tau)$ of the function $M(u)$ is continuous on its domain. Also suppose that $0 \leq t \leq 1 / 2$. Then, for any $\delta \in(0, \infty)$, there exists a unique number $h=h(\delta, t)$ for which $\left\|x_{t, h}\right\|_{\infty}=\delta$ and, for any $h>0$,

$$
\omega_{N}\left(D_{t},\left\|x_{t, h}\right\|_{\infty}\right)=\left|x_{t, h}^{\prime}(t)\right|
$$

Theorem 4. Suppose that $M(u)$ and $N(v)$ are complementary $N$-functions and the right derivative $p(\tau)$ of the function $M(u)$ is continuous on its domain. Then, for any $\delta \in(0, \infty)$, there exists a unique number $h=h(\delta, t)$ for which $\left\|x_{t, h}\right\|_{\infty}=\delta$ and, for any $h>0$,

$$
\omega_{N}\left(D,\left\|x_{t, h}\right\|_{\infty}\right)=\left\|x_{t, h}^{\prime}\right\|_{\infty}
$$

In the case $N(u)=u^{s}, 1<s<\infty$, we set $\omega_{s}(D, \delta):=\omega_{N}(D, \delta)$. Note that the function $\omega_{s}(D, \delta)$ can be expressed explicitly with respect to $\delta$ as

$$
\omega_{s}(D, \delta)= \begin{cases}\left(\frac{s^{\prime}+1}{s^{\prime}}\right)^{s^{\prime} /\left(s^{\prime}+1\right)}(2 \delta)^{1 /\left(s^{\prime}+1\right)}, & \delta \in\left(0, \frac{1}{2 s^{\prime}\left(s^{\prime}+1\right)^{1 / s^{\prime}}}\right] \\ 2 \delta+\frac{1}{\left(s^{\prime}+1\right)^{1 / s^{\prime}}}, & \delta \in\left[\frac{1}{2 s^{\prime}\left(s^{\prime}+1\right)^{1 / s^{\prime}}}, \infty\right)\end{cases}
$$

where $s^{\prime}=s /(s-1)$.

\section{APPLICATIONS}

As applications of the results obtained, we shall solve the Stechkin problem of approximating unbounded operators $D$ and $D_{t}, t \in[0,1]$, by linear bounded operators as well as the Kolmogorov problem for three numbers. Let us present the formulations of the corresponding problems. 
The Stechkin problem. The classical statement of the Stechkin problem is given, for example, in the book [4]. Suppose that $X$ and $Y$ are Banach spaces and $T: X \rightarrow Y$ is a linear unbounded operator with domain $D(T) \subset X$. Also suppose that $Q \subset D(T)$. By $\mathcal{L}(K), K>0$, we denote the set of all linear bounded operators $A: X \rightarrow Y$ whose norm is at most $K$. Then the quantity

$$
U(T ; A, Q):=\sup _{x \in Q}\|T x-A x\|_{Y}
$$

is the deviation of the operator $A$ from the operator $T$ for the set $Q$, and

$$
E_{K}(T ; Q):=\inf _{A \in \mathcal{L}(K)} U(T ; A, Q)
$$

is the value of the best approximation of the operator $T$ by the set of bounded operators $\mathcal{L}(K)$ for the set $Q$.

In [11], the following often-used lower bound

$$
E_{K}(T ; Q) \geq \sup _{\delta>0}(\omega(T, \delta)-K \delta), \quad K>0,
$$

was obtained for the value of the best approximation $E_{K}(T ; Q)$ via the modulus of continuity

$$
\omega(T, \delta):=\sup \left\{\|T x\|_{Y}: x \in Q,\|x\|_{X} \leq \delta\right\}
$$

of the operator $T$ for the set $Q$. A survey of known cases of the solution of the Stechkin problem as well as further references can be found, for example, in [12].

The solution of the Stechkin problem for the operators $D$ and $D_{t}, 0 \leq t \leq 1$, for the class $W_{M}^{*, 2}$ is given in the following theorem.

Theorem 5. Suppose that $M(u)$ and $N(v)$ are complementary $N$-functions and the right derivative $p(\tau)$ of the function $M(u)$ is continuous on its domain. Then, for any $0 \leq t \leq 1 / 2$,

$$
\begin{gathered}
E_{K}\left(D_{t} ; W_{N}^{*, 2}\right)= \begin{cases}+\infty, & 0<K<2, \\
\left\|g_{t, 2 / K}\right\|_{M}, & 2 \leq K<\infty,\end{cases} \\
E_{K}\left(D ; W_{N}^{*, 2}\right)= \begin{cases}+\infty, & 0<K<2, \\
\left\|g_{0,2 / K}\right\|_{M}, & 2 \leq K<\infty,\end{cases}
\end{gathered}
$$

where the function $g_{t, h}(\tau)$ is defined by relations (1.3) and (1.4).

The Kolmogorov problem. Suppose that $n \in \mathbb{N}, n \geq 3$. The Kolmogorov problem [13], [14] for $n$ numbers is to find necessary and sufficient conditions such that, for given positive numbers

$$
M_{\nu_{i}, p_{i}}, \quad 1 \leq p_{i} \leq \infty, \quad 1 \leq \nu_{i} \leq r, \quad i=1,2, \ldots, n,
$$

and a given class $X$ of smooth functions, there exists a function $f \in X$ for which

$$
\left\|f^{\left(\nu_{i}\right)}\right\|_{p_{i}}=M_{\nu_{i}, p_{i}} .
$$

The known cases of the solution of this problem and further references can be found, for example, in the book [4].

Theorem 6. Suppose that $M(u)$ and $N(v)$ are complementary $N$-functions and the right derivative $p(\tau)$ of the function $M(u)$ is continuous on its domain. Also suppose that $0 \leq t \leq 1 / 2$. Then, for positive numbers $M_{0}, M_{1}$, and $M_{2}$, there exists a function $x \in L_{N}^{*, 2}$ for which

$$
\|x\|_{\infty}=M_{0}, \quad\left\|x^{\prime}\right\|=M_{1}, \quad\left\|x^{\prime \prime}\right\|_{(N)}=M_{2}
$$

if and only if

$$
\frac{M_{1}}{M_{2}} \leq \omega_{N}\left(D, \frac{M_{0}}{M_{1}}\right) .
$$


A generalization of Theorem 6 is given in the following result.

Theorem 7. Suppose that $M(u)$ and $N(v)$ are complementary $N$-functions and the right derivative $p(\tau)$ of the function $M(u)$ is continuous on its domain. Also suppose that $0 \leq t \leq 1 / 2$. Then, for positive numbers $M_{0}, M_{1}$, and $M_{2}$, there exists a function $x \in L_{N}^{*, 2}$ for which

$$
\|x\|_{\infty}=M_{0}, \quad\left|x^{\prime}(t)\right|=M_{1}, \quad\left\|x^{\prime \prime}\right\|_{(N)}=M_{2}
$$

if and only if

$$
\frac{M_{1}}{M_{2}} \leq \omega_{N}\left(D_{t}, \frac{M_{0}}{M_{1}}\right)
$$

Remark 2. In the case of the classes $L_{s}, 1 \leq s<\infty$, Theorems 6 and 7 were obtained in [8].

\section{PROOFS}

Proof of Theorem 3. It is readily seen from Lemma 1 and Definitions (1.7) and (2.1) of the functions $x_{t, h}$ that, for any $0<h<\infty$,

$$
\omega_{N}\left(D_{t},\left\|x_{t, h}\right\|_{\infty}\right)=\left|x_{t, h}^{\prime}(t)\right|
$$

To conclude the proof of the theorem, it remains to verify that the function $\left\|x_{t, h}\right\|_{\infty}$ strictly increases with respect to $h$ and takes all positive values. To this end, note that the function $\omega_{N}\left(D_{t}, \delta\right)$ is strictly increasing. Indeed, suppose that $0<\delta_{1}<\delta_{2}$. Then, for $\varepsilon=\delta_{2}-\delta_{1}$, there exists a function $x \in W_{N}^{*, 2}$, $\|x\|_{\infty} \leq \delta_{1}$, such that

$$
\left|x^{\prime}(t)\right| \geq \omega_{N}\left(D_{t}, \delta_{1}\right)-\varepsilon
$$

Consider the function

$$
\bar{x}(u):=x(u)+2\left(\delta_{2}-\delta_{1}\right)\left(t-\frac{1}{2}\right) \operatorname{sign} x^{\prime}(t) .
$$

Obviously, $\bar{x} \in W_{N}^{*, 2},\|\bar{x}\|_{\infty} \leq \delta_{2}$ and

$$
\omega_{N}\left(D_{t}, \delta_{2}\right) \geq\left|\bar{x}^{\prime}(t)\right|=\left|x^{\prime}(t)\right|+2\left(\delta_{2}-\delta_{1}\right) \geq \omega_{N}\left(D_{t}, \delta_{1}\right)+\delta_{2}-\delta_{1}>\omega_{N}\left(D_{t}, \delta_{1}\right),
$$

which proves the assertion. Therefore, in view of relation (2.2), to verify the strict monotonicity of the function $\left\|x_{t, h}\right\|_{\infty}$, it is necessary and sufficient to verify the strict monotonicity of the function $\left|x_{t, h}^{\prime}(t)\right|$.

First, consider the case $h \in(0,2 t]$. Obviously, relation (1.6) can be rewritten as

$$
2 h \int_{0}^{1 / 2} N\left[p\left(\alpha_{h} u\right)\right] d u=1 .
$$

It is also easy to verify that

$$
\left|x_{t, h}^{\prime}(t)\right|=h \int_{0}^{1 / 2} p\left(\alpha_{h} u\right) d u .
$$

It follows from relation (4.1) for $\alpha_{h}$ that $\alpha_{h}$ decreases as $h$ increases, because the functions $p$ and $N$ increase on $[0, \infty)$. Therefore, the fact that $\left|x_{t, h}^{\prime}(t)\right|$ strictly increases in $h$, will be proved as soon as we prove that the function

$$
\mu(a):=\frac{\int_{0}^{1 / 2} p(a u) d u}{\int_{0}^{1 / 2} N[p(a u)] d u}
$$

decreases for $a>0$. The latter is obvious, because, in view of the convexity of the function $N(v)$ and the fact that $N(0)=0$, we have the inequality

$$
\frac{N(u)}{u} \leq \frac{N(v)}{v}
$$


for all $0<u \leq v$ and, therefore,

$$
\mu^{\prime}(a)=\frac{p(a / 2) \int_{0}^{1 / 2} N[p(a u)] d u-N[p(a / 2)] \int_{0}^{1 / 2} p(a u) d u}{\left(\int_{0}^{1 / 2} N[p(a u)] d u\right)^{2}} \leq 0 .
$$

Let us now verify that

$$
\lim _{h \rightarrow 0}\left\|x_{t, h}\right\|_{\infty}=0 .
$$

Note that $\alpha_{h} \rightarrow+\infty$ as $h \rightarrow 0$. Therefore, to prove (4.3), by relation (4.1) it suffices to prove that $\mu(a) \rightarrow 0$ as $a \rightarrow+\infty$. By L'Hospital's rule, we have

$$
\lim _{a \rightarrow+\infty} \mu(a)=\lim _{a \rightarrow+\infty} \frac{p(a / 2)}{N[p(a / 2)]}=\lim _{b \rightarrow+\infty} \frac{b}{N(b)},
$$

because $p(a) \rightarrow+\infty$ as $a \rightarrow+\infty$. However, for any $b>0$, the following inequality holds:

$$
N(b) \geq \int_{b / 2}^{b} q(u) d u \geq \frac{b}{2} q\left(\frac{b}{2}\right) .
$$

Therefore,

$$
0 \leq \lim _{a \rightarrow+\infty} \mu(a)=\lim _{b \rightarrow+\infty} \frac{b}{N(b)} \leq \lim _{b \rightarrow+\infty} \frac{2}{N(b / 2)}=0
$$

because $q(b) \rightarrow+\infty$ as $b \rightarrow+\infty$. The last relation implies the limit equality (4.3).

Thus, the function $\left\|x_{t, h}\right\|_{\infty}$ strictly increases and takes all the values from zero to $\left\|x_{t, 2 t}\right\|_{\infty}$, as $h$ ranges over the half-interval $(0,2 t]$.

Further, consider the case $h \in[2 t, 1]$. Now relation (1.6) can be rewritten as:

$$
\frac{h}{\alpha_{h}} \int_{0}^{\alpha_{h} t / h} N[p(u)] d u+\frac{h}{\alpha_{h}} \int_{0}^{\alpha_{h}(1-t / h)} N[p(u)] d u=1 .
$$

In addition,

$$
\left|x_{t, h}^{\prime}(t)\right|=\frac{h}{\alpha_{h}} \int_{0}^{\alpha_{h}(1-t / h)} p(u) d u .
$$

In order to prove that $\left|x_{t, h}^{\prime}(t)\right|$ strictly increases in $h$, we differentiate the function $F(h):=\left|x_{t, h}^{\prime}(t)\right|$ with respect to $h$ :

$$
\begin{gathered}
F^{\prime}(h)=\frac{1}{\alpha_{h}} \int_{0}^{\alpha_{h}(1-t / h)} p(u) d u-\frac{h \alpha_{h}^{\prime}}{\alpha_{h}^{2}} \int_{0}^{\alpha_{h}(1-t / h)} p(u) d u \\
+\left[\frac{h \alpha_{h}^{\prime}}{\alpha_{h}}\left(1-\frac{t}{h}\right)+\frac{t}{h}\right] p\left(\alpha_{h}\left(1-\frac{t}{h}\right)\right) .
\end{gathered}
$$

Differentiating relation (4.4) we obtain

$$
\begin{aligned}
& \frac{h \alpha_{h}^{\prime}}{\alpha_{h}}\left(1-t N\left[p\left(\alpha_{h} \frac{t}{h}\right)\right]-(h-t) N\left[p\left(\alpha_{h}\left(1-\frac{t}{h}\right)\right)\right]\right) \\
& \quad=1-t N\left[p\left(\alpha_{h} \frac{t}{h}\right)\right]+t N\left[p\left(\alpha_{h}\left(1-\frac{t}{h}\right)\right)\right] .
\end{aligned}
$$

Substituting this relation for $\alpha_{h}^{\prime}$ into the expression for the derivative $F^{\prime}(h)$, we find that the assertion that the derivative $F^{\prime}(h)$ is positive is equivalent to that of the validity of the inequality

$$
\frac{N\left[p\left(\alpha_{h}(1-t / h)\right)\right]}{p\left(\alpha_{h}(1-t / h)\right)} \int_{0}^{\alpha_{h}(1-t / h)} p(u) d u \geq \frac{\alpha_{h}}{h}-\frac{t \alpha_{h}}{h} N\left[p\left(\alpha_{h}\left(1-\frac{t}{h}\right)\right)\right] .
$$


In view of (4.2) and (4.4), inequality (4.5) is a consequence of the inequality

$$
\frac{t \alpha_{h}}{h} N\left[p\left(\alpha_{h}\left(1-\frac{t}{h}\right)\right)\right] \geq \int_{0}^{\alpha_{h} t / h} N[p(u)] d u .
$$

The last inequality always holds, because $N(v)$ is an increasing function and, in the case under consideration, $t / h \leq 1-t / h$. Thus, the function $\left\|x_{t, h}\right\|_{\infty}$ increases in $h$ on $[h, 1]$ and, takes all the values from $\left\|x_{t, 2 t}\right\|_{\infty}$ to $\left\|x_{t, 1}\right\|_{\infty}$, because it is continuous in $h$.

Finally, consider the case in which $h \geq 1$. Obviously, in that case,

$$
\left\|x_{t, h}\right\|_{\infty}=\frac{h+1}{2}\left\|x_{t, 1}\right\|_{\infty}
$$

Therefore, $\left\|x_{t, h}\right\|_{\infty}$ increases and takes all the values from $\left\|x_{t, 1}\right\|_{\infty}$ to $+\infty$.

Proof of Theorem 4. Obviously, by Theorem 2, we have

$$
\omega_{N}(D, \delta)=\omega_{N}\left(D_{0}, \delta\right)
$$

for any $\delta>0$, which concludes the proof.

Proof of Theorem 5. To find the lower bound of the quantity $E_{K}\left(D_{t} ; W_{N}^{*, 2}\right)$, we use inequality (3.1):

$$
E_{K}\left(D_{t} ; W_{N}^{*, 2}\right) \geq \sup _{\delta>0}\left(\omega_{N}\left(D_{t}, \delta\right)-K \delta\right) .
$$

In view of Theorem 3 , the last inequality can be rewritten as

$$
E_{K}\left(D_{t} ; W_{N}^{*, 2}\right) \geq \sup _{h>0}\left(\left|x_{t, h}^{\prime}(t)\right|-K\left\|x_{t, h}\right\|_{\infty}\right) .
$$

Now consider the case $K \geq 2$. Using inequality (4.6) and recalling the construction of the functions $x_{t, h}(u)$ for $h \in[0,1]$ (see (1.7)), we obtain

$$
E_{K}\left(D_{t} ; W_{N}^{*, 2}\right) \geq \sup _{h \in[0,1]}\left(\left(\frac{2}{h}-K\right)\left\|x_{t, h}\right\|_{\infty}+\left\|g_{t, h}\right\|_{M}\right) \geq\left\|g_{t, \frac{2}{K}}\right\|_{M} .
$$

Let us define the operator $S_{t, K}: L_{\infty} \rightarrow \mathbb{R}$ as follows:

$$
S_{t, K}(x):= \begin{cases}\frac{K[x(2 / K)-x(0)]}{2}, & K \in\left[2, \frac{1}{t}\right], \\ \frac{K[x(t+1 / K)-x(t-1 / K)]}{2}, & K \in\left[\frac{1}{t},+\infty\right) .\end{cases}
$$

Obviously,

$$
\left|S_{t, K}(x)\right| \leq K\|x\|_{\infty},
$$

whence the norm of the operator $S_{t, K}$ is at most $K$. Therefore,

$$
\begin{aligned}
E_{K}\left(D_{t} ; W_{N}^{*, 2}\right) & \leq U\left(D_{t} ; S_{t, K}, W_{N}^{*, 2}\right)=\sup _{\left\|x^{\prime \prime}\right\|_{(N)} \leq 1}\left|x^{\prime}(t)-S_{t, K}(x)\right| \\
& =\sup _{\left\|x^{\prime \prime}\right\|_{(N)} \leq 1}\left|\int_{0}^{1} g_{t, 2 / K}(\tau) x^{\prime \prime}(\tau) d \tau\right|=\left\|g_{t, 2 / K}\right\|_{M} .
\end{aligned}
$$

Comparing inequalities (4.7) and (4.9), we obtain the required equality (3.2) for $K \geq 2$.

It remains to consider the case $K \in(0,2)$. Then, from inequality (4.6), we obtain

$$
E_{K}\left(D_{t} ; W_{N}^{*, 2}\right) \geq \sup _{h \geq 1}\left(\left|x_{t, h}^{\prime}(t)\right|-K\left\|x_{t, h}\right\|_{\infty}\right)
$$


By the construction of the functions $\varphi_{t, h}$ for $h \geq 1$ (see $(2.1)$ ), we have the relations

$$
\left\|x_{t, h}\right\|_{\infty}=\frac{h+1}{2}\left\|x_{t, 1}\right\|_{\infty} \quad \text { and } \quad\left|x_{t, h}^{\prime}(t)\right|=\left|x_{t, 1}^{\prime}(t)\right|+(h-1)\left\|x_{t, 1}\right\|_{\infty} .
$$

Substituting these equalities into inequality (4.10), we obtain

$$
E_{K}\left(D_{t} ; W_{N}^{*, 2}\right) \geq\left|x_{t, 1}^{\prime}(t)\right|-\left(1+\frac{K}{2}\right)\left\|x_{t, 1}\right\|_{\infty}+\sup _{h \geq 1}\left(1-\frac{K}{2}\right)\left\|x_{t, 1}\right\|_{\infty}=+\infty .
$$

Therefore, equality (3.2) is finally proved.

To obtain the second of the equalities in Theorem 5 , we now need to use Theorem 4 , the intermediate operator $S_{K}: L_{\infty} \rightarrow L_{\infty}$, given by the rule

$$
S_{K} x(t)=S_{t, K}(x), \quad x \in L_{\infty},
$$

and inequality (1.11).

Proof of Theorem 6. The necessity of condition (3.3) is obvious because of the definition of the modulus of continuity $\omega_{N}(D, \delta)$. In order to prove the sufficiency of condition (3.3), we note that the continuity of the modulus of continuity $\omega_{N}\left(D_{0}, \delta\right)$ was verified in the proof of Theorem 3 and hence also, by Theorem 4 , that of the modulus of continuity $\omega_{N}(D, \delta)$. Therefore, for all numbers $M_{0}, M_{1}$, and $M_{2}$ satisfying conditions (3.3), there exists a number $h \in\left(0, M_{0} / M_{2}\right)$ such that

$$
\omega_{N}\left(D,\left\|x_{0, h}\right\|\right)=\frac{M_{1}}{M_{2}}
$$

Consider the function

$$
x(u):=M_{2}\left(x_{0, h}(u)+\frac{M_{0}}{M_{2}}-h\right) .
$$

It is simple to verify that $\left\|x^{\prime \prime}\right\|_{(N)}=M_{2}$,

$$
\left\|x^{\prime}\right\|_{\infty}=M_{2}\left\|x_{0, h}\right\|_{\infty}=M_{2} \omega_{N}\left(D,\left\|x_{0, h}\right\|\right)=M_{1},
$$

and $\|x\|_{\infty}=M_{0}$, which proves the assertion.

Proof of Theorem 7. The proof is similar.

\section{REFERENCES}

1. M. A. Krasnosel'skii and Ya. B. Rutitskii, Convex Functions and Orlicz Spaces (Fizmatgiz, Moscow, 1958) [in Russian].

2. E. Landau, "Einige Ungleichungen fur zweimal differenzierbare Funktionen," Proc. London Math. Soc. (2) $13(1), 43-49(1913)$.

3. M. K. Kwong and A. Zettl, Norm Inequalities for Derivatives and Differences, in Lecture Notes in Math. (Springer-Verlag, Berlin, 1992), Vol. 1536 [in Russian].

4. V. F. Babenko, N. P. Korneichuk, V. A. Kofanov, and S. A. Pichugov, Inequalities for Derivatives and Their Applications (Naukova Dumka, Kiev, 2003) [in Russian].

5. A. Yu. Shadrin, "To the Landau-Kolmogorov problem on a finite interval," in Open Problems in Approximation Theory (SCT Publ., Singapore, 1994), pp. 192-204 [in Russian].

6. A. Pinkus, "Some extremal properties of perfect splines and the pointwise Landau problem on the finite interval," J. Approx. Theory 23 (1), 37-64 (1978).

7. Yu. V. Babenko, "Sharp Landau-type inequalities for functions with second derivatives from Orlicz spaces," Vestnik Dnepropetrovsk. Univ., No. 2, 18-22 (2000).

8. V. I. Burenkov and V. A. Gusakov, "On sharp constants in inequalities for the modulus of a derivative," in Trudy Mat. Inst. Steklov, Vol. 243: Function Spaces, Approximations, and Differential Equations, Collection of papers on the occasion of the 70th anniversary of the Corresponding Member of the Russian Academy of Sciences O. V. Besov (Nauka, Moscow, 2003), pp. 104-126 [Proc. Steklov Inst. Math. 243, 98-119(2003)].

9. N. P. Korneichuk, V. F. Babenko, and A. A. Ligun, Extremal Properties of Polynomials and Splines (Naukova Dumka, Kiev, 1992) [in Russian]. 
10. Yu. V. Babenko, "Pointwise inequalities of Landau-Kolmogorov type for functions defined on a finite segment," Ukrain. Mat. Zh. 53 (2), 238-243 (2001) [Ukrainian Math. J. 53 (2), 270-275 (2001)].

11. S. B. Stechkin, "Best approximation of linear operators," Mat. Zametki 1 (2), 137-148 (1967) [Math. Notes $1(2), 91-99(1968)]$.

12. V. V. Arestov, "Approximation of unbounded operators by bounded operators and related extremal problems," Uspekhi Mat. Nauk 51 (6), 89-124 (1996) [Russian Math. Surveys 51 (6), 1093-1126 (1996)].

13. A. N. Kolmogorov, "On inequalities between the upper bounds for the successive derivatives of an arbitrary function on the infinite interval," in Uchen. Zapiski Moskov. Gos. Univ. (Izd. Moskov. Univ., Moscow, 1939), Vol. 30, pp. 3-16 [in Russian].

14. A. N. Kolmogorov, "On inequalities between the upper bounds for the successive derivatives of an arbitrary function on the infinite interval," in Selected works. Mathematics and mechanics (Nauka, Moscow, 1985), pp. 252-263 [in Russian]. 